\title{
Nonlinear response of single-molecule magnets: Field-tuned quantum-to-classical crossovers
}

\author{
R. López-Ruiz, F. Luis,* A. Millán, C. Rillo, D. Zueco, and J. L. García-Palacios \\ Instituto de Ciencia de Materiales de Aragón y Departamento de Física de la Materia Condensada, CSIC-Universidad de Zaragoza, \\ E-50009 Zaragoza, Spain
}

(Received 12 June 2006; published 11 January 2007)

\begin{abstract}
Quantum nanomagnets can show a field dependence of the relaxation time very different from their classical counterparts, due to resonant tunneling via excited states (near the anisotropy barrier top). The relaxation time then shows minima at the resonant fields $H_{n} \propto n D$ at which the levels at both sides of the barrier become degenerate ( $D$ is the anisotropy constant). We showed that in $\mathrm{Mn}_{12}$, near zero field, this yields a contribution to the nonlinear susceptibility that makes it qualitatively different from the classical curves [Phys. Rev. B 72, 224433 (2005)]. Here we extend the experimental study to finite dc fields showing how the bias can trigger the system to display those quantum nonlinear responses, near the resonant fields, while recovering a classical-like behaviour for fields between them. The analysis of the experiments is done with heuristic expressions derived from simple balance equations and calculations with a Pauli-type quantum master equation.
\end{abstract}

DOI: 10.1103/PhysRevB.75.012402

PACS number(s): 75.45. $+\mathrm{j}, 75.50 . \mathrm{Xx}, 75.50 . \mathrm{Tt}, 75.40 . \mathrm{Gb}$

\section{INTRODUCTION}

Single-molecule magnets are metal-organic clusters containing a magnetic core surrounded by a shell of organic ligands which isolates the clusters from one another (see, e.g., Refs. 1). The most studied is $\mathrm{Mn}_{12}$, whose core contains eight $\mathrm{Mn}^{3+}$ and four $\mathrm{Mn}^{4+}$ ions strongly coupled via superexchange interactions. This gives a ground state spin $S=10$, while the large Jahn-Teller distortion on the $\mathrm{Mn}^{3+}$ sites leads to a strong uniaxial anisotropy. The energy levels have a bistable structure $\varepsilon_{m} \sim-D m^{2}$ (at zero field) with an energy barrier $U=\varepsilon_{0}-\varepsilon_{S} \simeq 70 \mathrm{~K}$ to be overcome for the spin reversal. At low temperatures, these systems show the typical behaviors of superparamagnets, such as blocking or hysteresis, yet at a much smaller scale of size. In addition, they form molecular crystals in which all molecules are nearly identical and, in the case of $\mathrm{Mn}_{12}$ acetate, have their anisotropy axes $z$ parallel to the crystallographic $c$ axis.

These properties make of molecular magnets model systems to investigate whether quantum phenomena, like tunneling, survive in mesoscopic systems. ${ }^{2}$ As is well known, tunneling probabilities decrease exponentially with the height of the barrier to be tunneled through (a height that grows with the system size). ${ }^{3}$ At the same time, external perturbations can induce decoherence that degrades the quantum behavior. ${ }^{4,5}$ Furthermore, an external magnetic field $H_{z}$ detunes energetically the initial and final states for tunneling [i.e., those having $+m$ and $-(m+n)$ spin projections along $z$ ]. Actually, many experiments have shown that tunneling takes place at those fields where states of opposite orientation are degenerate, $H_{n} \simeq n H_{1}\left(n=0,1,2, \ldots\right.$ with $H_{1}$ $=2 g \mu_{\mathrm{B}} D \simeq 4200$ Oe in $\mathrm{Mn}_{12}$ ), whereas it is suppressed for intermediate fields. $^{6-8}$

Such a resonant tunneling enables the spins to approach faster their equilibrium state, giving rise to steps in the hysteresis loops around $H_{z}=H_{n}, 8,9$ and to maxima in the linear dynamical susceptibility $\chi_{1}$. In our previous work, ${ }^{10,11}$ we found that resonant tunneling at $H_{z}=0$ induces an extra contribution to the nonlinear response $\chi_{3}$, making it larger (in magnitude) than the equilibrium one and having peaks re- versed with respect to the classical predictions. ${ }^{12,13}$ In Refs. 10 and 11 the dependence of the response on temperature, frequency, and orientation at zero field was studied. In this Brief Report we show how the tunneling contribution to the nonlinear response can be switched on and off by varying an external field, tuning and breaking the resonances successively.

\section{EXPERIMENTAL DETAILS}

Single crystals of $\mathrm{Mn}_{12}$ acetate were grown following the same procedure described in Ref. 11. In order to increase the signal, the magnetic measurements were done on a collection of oriented and glued crystals. In our previous experiments ${ }^{10,11}$ we extracted the zero-field $\chi_{3}(\omega)$ by fitting the dc field-dependent $\chi(\omega)$ to a parabola. Clearly, this

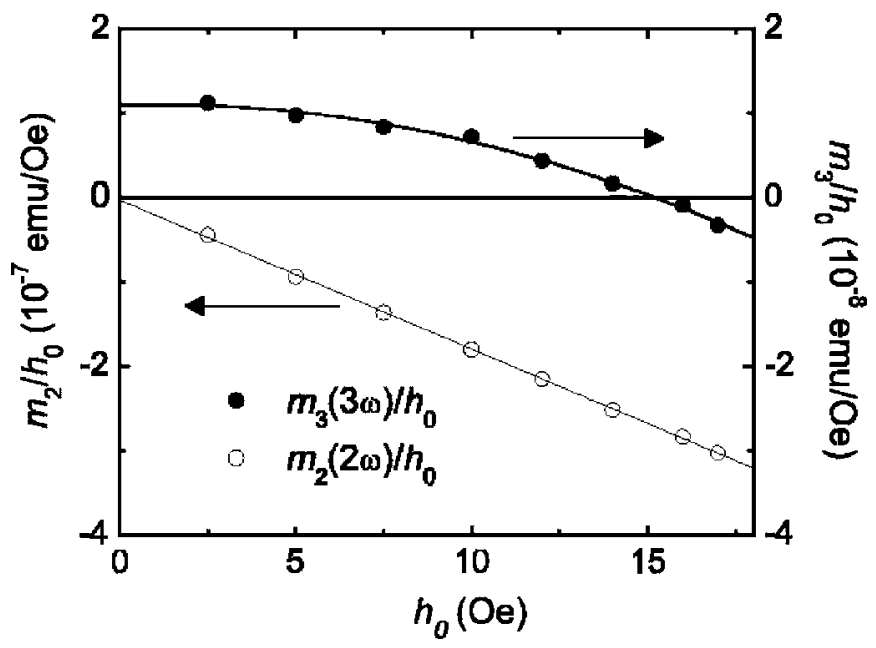

FIG. 1. Illustration of the method employed for measuring the nonlinear susceptibilities (here $T=8 \mathrm{~K}, H_{z}=100 \mathrm{Oe}$, and $\omega / 2 \pi$ $=2 \mathrm{kHz}) \cdot \chi_{2}(2 \omega)$ and $\chi_{3}(3 \omega)$ are obtained, respectively, from the slope and the quadratic coefficient of the second $m_{2}(2 \omega) / h_{0}$ and third $m_{3}(3 \omega) / h_{0}$ harmonics of the output signal, measured as a function of the ac field amplitude $h_{0}$. 
method is not applicable to study how $\chi_{3}$ depends on the external magnetic field itself. For this reason, in the present experiments we resorted to the more traditional method of measuring nonlinear susceptibilities by detecting the different harmonics $\chi_{2}(2 \omega)$ and $\chi_{3}(3 \omega)$ of the response. In the absence of bias field one has $\chi_{2} \equiv 0$ (see below). A nonzero $H_{z}$, however, makes $\chi_{2}$ the leading nonlinear term, and we will mainly focus on it.

The fields, both dc and ac, were applied parallel to the common anisotropy direction $z$ of the clusters. We employed the susceptibility option of a commercial multipurpose measuring platform (physical properties measuring system, PPMS) which uses a conventional inductive method. It enables applying ac fields of amplitude $h_{0} \leq 17 \mathrm{Oe}$, and selective detection of several harmonics of the exciting frequency $\omega / 2 \pi<10 \mathrm{kHz}$. To separate the intrinsic nonlinear response of the sample from the possible contamination due to nonperfect harmonicity of the exciting ac coil, we measured the output signals $m_{2}(2 \omega) / h_{0}$ and $m_{3}(3 \omega) / h_{0}$ at several $h_{0}$. This gives the sought-for intrinsic contributions $\chi_{2}$ and $\chi_{3}$ as the terms proportional to $h_{0}$ and $h_{0}^{2}$, respectively. An example of this procedure is shown in Fig. 1.

\section{RESULTS AND MODELIZATION}

The so-measured linear and nonlinear susceptibilities of $\mathrm{Mn}_{12}$ at $T=8 \mathrm{~K}$ are shown in Fig. 2. The frequencydependent $\chi_{1}$ shows maxima near the resonant fields, $H_{0}=0$, $H_{1}$, and $H_{2}$, where it approaches the equilibrium $\chi_{1}^{\text {eq }}$. We also display [Fig. 2(b)] the second and third harmonic components measured at $\omega / 2 \pi=2 \mathrm{kHz}$. (The high noise-to-signal ratio prevented us from obtaining reliable $\chi_{3}$ data for $H_{z}$ $>1$ kOe.) The equilibrium nonlinear susceptibilities, also shown, were obtained by differentiating $\chi_{1}^{\mathrm{eq}}$, measured at the lowest frequency $\omega / 2 \pi=1 \mathrm{~Hz}$.

We clearly see that the magnitudes of the harmonics increase in the neighborhood of the resonant fields $H_{0}=0, H_{1}$, and $\mathrm{H}_{2}$, where states of opposite $S_{z}$ are degenerate and the tunnel channels open. In addition, in contrast to the behavior of $\chi_{1}$, both $\chi_{2}$ and $\chi_{3}$ become, near $H_{0}$, larger than $\chi_{2}^{\mathrm{eq}}$ and $\chi_{3}^{\text {eq }}$. Thus, when resonant tunneling sets in, the multiharmonic response of these molecular clusters is enhanced.

In order to understand these results we have derived simple expressions for the susceptibilities. This was done by solving, as in Ref. 13, a system of balance equations for the net population of the two anisotropy-potential wells:

$$
\begin{gathered}
\chi_{1}(\omega)=\chi_{1}^{\mathrm{eq}} \frac{1}{1+i \omega \tau} \quad \chi_{2}(2 \omega)=\chi_{2}^{\mathrm{eq}} \frac{1}{1+2 i \omega \tau}-\chi_{1}^{\mathrm{eq}} \frac{i \omega \tau^{\prime}}{(1+i \omega \tau)(1+2 i \omega \tau)} \\
\chi_{3}(3 \omega)=\chi_{3}^{\mathrm{eq}} \frac{1}{1+3 i \omega \tau}-\chi_{1}^{\mathrm{eq}} \frac{\frac{1}{2} i \omega \tau^{\prime \prime}}{(1+i \omega \tau)(1+3 i \omega \tau)}-\chi_{2}^{\mathrm{eq}} \frac{2 i \omega \tau^{\prime}}{(1+2 i \omega \tau)(1+3 i \omega \tau)}-\chi_{1}^{\mathrm{eq}} \frac{2 i\left(\omega \tau^{\prime}\right)^{2}}{(1+i \omega \tau)(1+2 i \omega \tau)(1+3 i \omega \tau)} .
\end{gathered}
$$

The equilibrium $\chi_{k}^{\mathrm{eq}}=\left(d^{k} M_{z} / d H_{z}^{k}\right) / k$ ! are the derivatives of the magnetization curve, while $\tau, \tau^{\prime}$, and $\tau^{\prime \prime}$ are the relaxation time and its corresponding field derivatives (all evaluated at the working field $H_{z}$ ). At $H_{z}=0$ we have $\chi_{2}^{\mathrm{eq}} \equiv 0$ [since $M_{z}\left(H_{z}\right)=-M_{z}\left(-H_{z}\right)$ ] as well as $\tau^{\prime} \equiv 0$ [from $\tau\left(H_{z}\right)=\tau\left(-H_{z}\right)$ ]. Then $\chi_{2}(2 \omega) \rightarrow 0$ as $H_{z} \rightarrow 0$, while in $\chi_{3}$ the last two terms vanish. Therefore these equations extend the expressions of Ref. 13 to nonzero bias fields.

The first Eq. (1) gives the ratio $\chi_{1} / \chi_{1}^{\mathrm{eq}}<1$. In addition $\chi_{1}$ depends, via the product $\omega \tau$, on how far the spins are from thermal equilibrium. By contrast, the nonlinear susceptibilities $\chi_{2}$ and $\chi_{3}$ include also terms depending on $\tau^{\prime}$ and $\tau^{\prime \prime}$, i.e., on how sensitive $\tau$ is to changes of $H_{z}$. As a result, the relaxation time does not simply "renormalize frequency," as occurs with $\chi_{1}$, but it modifies the magnitudes of the nonlinear responses. This effect is missed in modelizations of the nonlinear susceptibility that fail to include the field derivatives of $\tau \cdot{ }^{15}$ As we discuss next, extending our arguments at $H_{z}=0$ of Refs. 10 and 11, it is this property that makes the quantum $\chi_{2}$ and $\chi_{3}$ qualitatively different from the classical ones.

According to Eq. (1), the relaxation time of the magnetic clusters can be estimated from $\chi_{1}$ as $\chi_{1}^{\prime \prime} / \omega \chi_{1}^{\prime}$ where $\chi_{1}^{\prime}$ and $\chi_{1}^{\prime \prime}$ are the real and imaginary components of the first harmonic. This $\tau$ is shown in the inset of Fig. 2. The data show minima at the resonant fields, in contrast with the monotontic behavior of $\tau$ in classical spins. At zero field and finite temperatures, $\mathrm{Mn}_{12}$ spins are able to tunnel between those excited magnetic states $(m \sim 2-4)$ for which this process is not blocked by the internal bias caused by dipolar and hyperfine interactions. ${ }^{16}$ This results in an effective barrier reduced by a few magnetic levels, say $U \sim \varepsilon_{ \pm 3}-\varepsilon_{ \pm S}$, so that the thermoactivated relaxation gets faster $(\delta U \sim 4 \mathrm{~K})$. Tunneling is, however, suppressed as soon as the external bias $\xi_{m}$ $=2 g \mu_{\mathrm{B}} m H_{z}$ exceeds the tunnel splitting $\Delta_{m}$, slowing down the relaxation (the full barrier has to be overcome). As a result, $\tau$ is minimum at zero field, whence $\tau^{\prime \prime}>0$, while $\tau^{\prime}$ changes sign from negative to positive.

The same features are repeated every time the field brings magnetic levels again into resonance $H_{n}=n \times\left(2 g \mu_{\mathrm{B}}\right) D$. Therefore, tunneling becomes, at any crossing field, an additional source of nonlinear response via $\tau^{\prime}$ and $\tau^{\prime \prime}$. In addition, accounting for the signs of the $\tau$ derivatives and Eqs. (1) and (2), one sees that the sign of the nonlinear susceptibilities can be reversed with respect to the classical ones. [In the classical model $\tau$ decreases monotonically with increasing field ${ }^{14}$ 


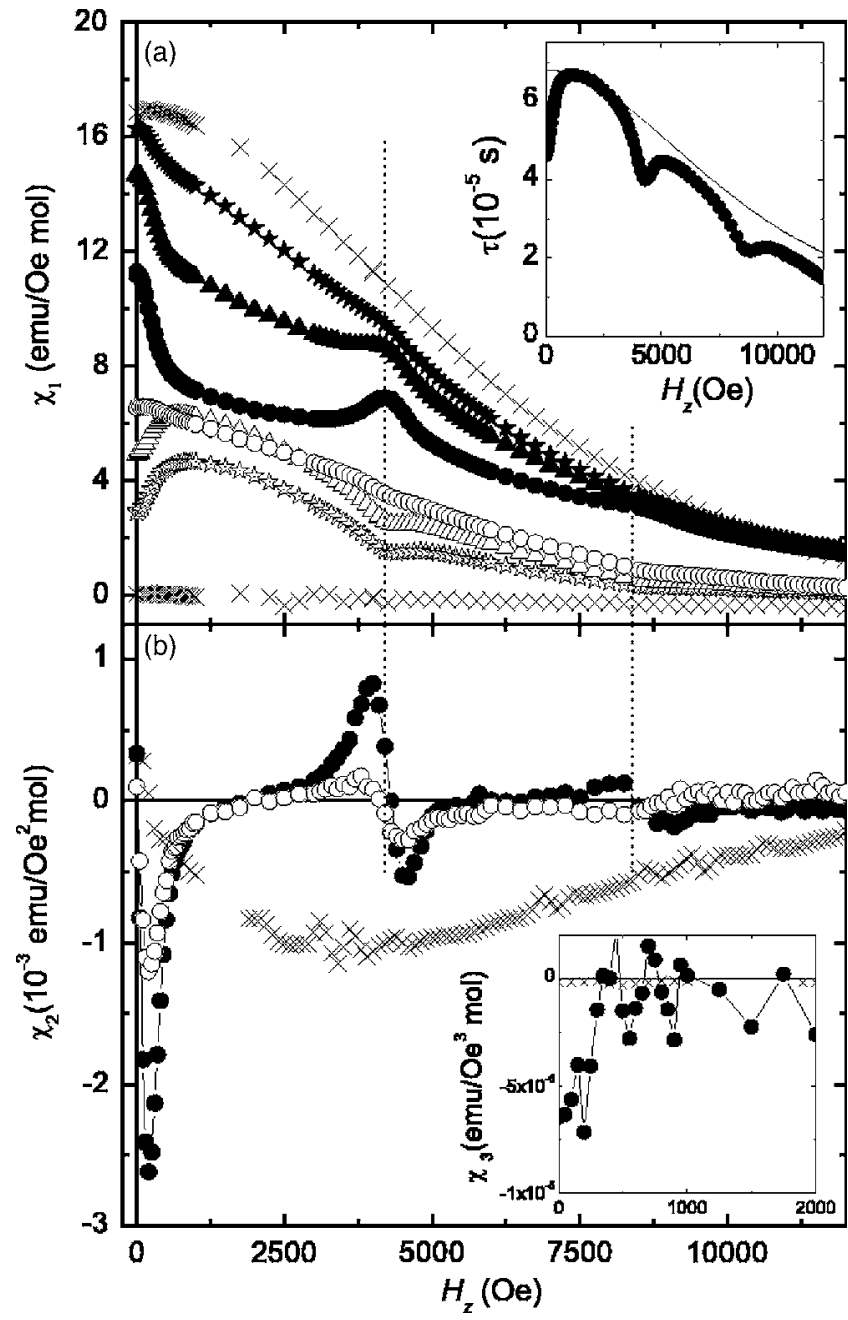

FIG. 2. (a) Linear susceptibility of $\mathrm{Mn}_{12}$ measured at $T=8 \mathrm{~K}$ versus a magnetic field applied parallel to the anisotropy axis. $\times$, $\omega / 2 \pi=1 \mathrm{~Hz}$ ( equilibrium);, $500 \mathrm{~Hz} ; \triangle, 1 \mathrm{kHz} ; \bullet, 2 \mathrm{kHz}$. Solid symbols, real parts; open symbols, imaginary parts. The inset shows the relaxation time $\tau$, as obtained from $\chi_{1}^{\prime \prime} / \omega \chi_{1}^{\prime}$ [see Eq. (1)] as well as calculated for classical spins (line) (Ref. 14). (b) Second harmonic susceptibility measured at the same temperature. - and $\circ$, $\chi_{2}^{\prime}(2 \omega)$ and $\chi_{2}^{\prime \prime}(2 \omega)$ at $2 \mathrm{kHz} ; \times$, equilibrium $\chi_{2}^{\mathrm{eq}}=\left(d \chi_{1}^{\mathrm{eq}} / d H_{z}\right) / 2$. Inset: $\bullet, \chi_{3}^{\prime}(3 \omega)$ at $2 \mathrm{kHz} ; \times, \chi_{3}^{\mathrm{eq}}=\left(d^{2} \chi_{1}^{\mathrm{eq}} / d H_{z}^{2}\right) / 6$. The dotted vertical lines mark the resonant fields $H_{1} \simeq 4200$ Oe and $H_{2}=2 H_{1}$.

(inset of Fig. 2), giving $\tau^{\prime \prime}<0$ and $\tau^{\prime}<0$ for any $H_{z}$; the same occurs in a quantum thermoactivation model not including the possibility of tunneling. $\left.{ }^{17,18}\right]$

It is interesting that both behaviors can be obtained in our case just by varying the external field. For fields between resonances, tunneling becomes blocked for all states and the spins reverse by thermal activation over the total ("classical") energy barrier. But when a crossing field is approached, the strong nonlinearity of $\tau$ shows up with its characteristic contribution to the nonlinear susceptibilities via $\tau^{\prime}$ and $\tau^{\prime \prime}$.

To confirm this interpretation we have computed the nonlinear responses from Eqs. (1) and (2) but incorporating the relaxation time obtained by solving a Pauli quantum master equation (as in Ref. 16). The results (Fig. 3) show that the quantum contribution to $\chi_{2}(2 \omega)$ is dominant near the reso-

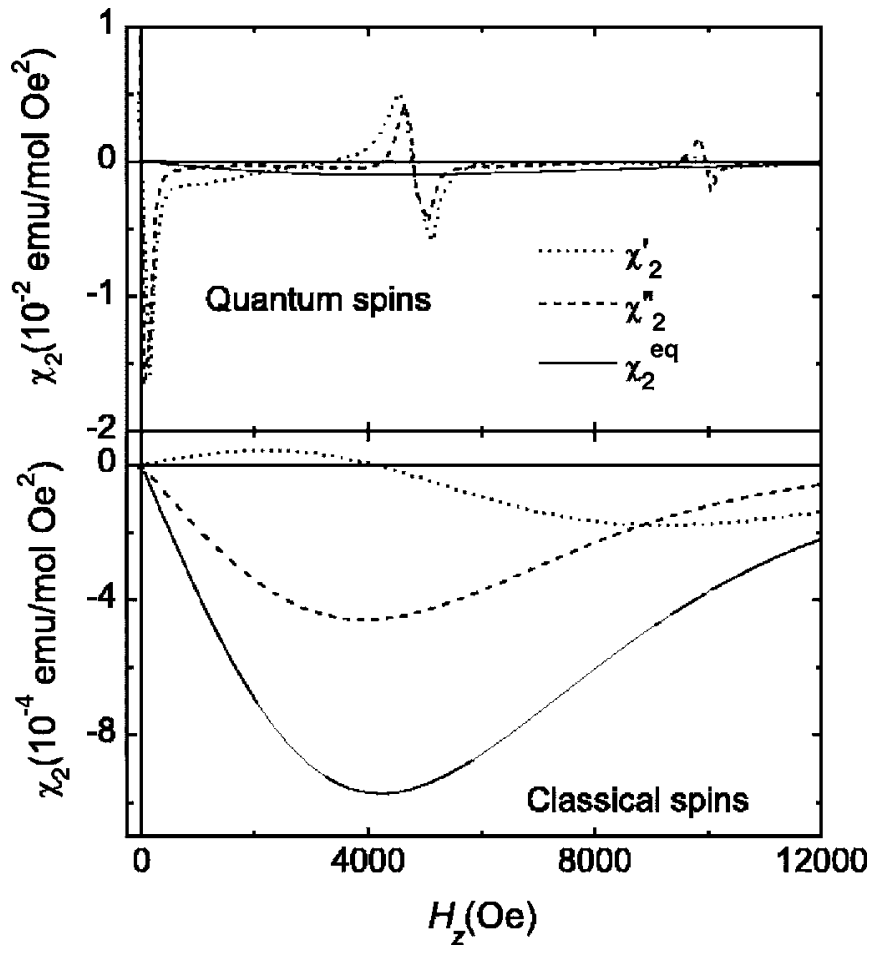

FIG. 3. Theoretical calculations of $\chi_{2}(2 \omega)$ [Eq. (2)] for quantum and classical spins. In the latter case, we used Brown's classical formula for $\tau$ (Ref. 14) and in the former $\tau$ was calculated by solving Pauli's quantum master equation, as described in Ref. 16. Notice the difference in the $\chi_{2}$ axis scale (the solid equilibrium curve is the same in both panels).

nances. This is due to the smallness of the tunnel splitting of the relevant states for our $\mathrm{Mn}_{12}$ sample: $\Delta_{4} \sim 2 \times 10^{-2} \mathrm{~K}$ and $\Delta_{2} \sim 7 \times 10^{-1} \mathrm{~K}$. This means that the fields required to block tunnel via these levels, albeit relatively small ( $\sim 20$ Oe and $1000 \mathrm{Oe}$, respectively), give rise to relatively large changes in $\tau$, and hence large $\tau^{\prime}$ and $\tau^{\prime \prime}$. In the classical regime, by contrast, the scale of change of $\tau$ is determined by the anisotropy field $H_{\mathrm{a}} \sim(2 S-1) H_{1}$. As this is very large in $\mathrm{Mn}_{12}$ $(\simeq 95 \mathrm{kOe})$, one has a comparatively slow decrease of $\tau$ with $H_{z}$. This correspond to small values of the derivatives $\tau^{\prime}$ and $\tau^{\prime \prime}$ and in turn of the classical (nontunneling) nonlinear susceptibilities.

It is also worth mentioning that the tunnel splittings, which determine the width of the $\tau$ vs $H_{z}$ resonances, are further broadened by dipolar and hyperfine interactions. ${ }^{16}$ In fact, the master-equation calculations tell us that tunneling via lower-lying states would give rise to enormous spikes in $\chi_{2}\left(\Delta_{10} \sim 7 \times 10^{-10} \mathrm{~K}\right.$ for the ground state). However, these peaks are also very fragile, being easily suppressed by environmental bias fields and therefore not observed experimentally.

\section{SUMMARY AND CONCLUSIONS}

We have studied experimentally the nonlinear susceptibilities of a $\mathrm{Mn}_{12}$ acetate molecular magnet in the presence of a longitudinal field $H_{z}$. The standard method of measuring 
the harmonics of the response to an oscillating field $h_{0} \cos (\omega t)$ has been employed. By using several amplitudes $h_{0}$ we managed to isolate the genuine nonlinear susceptibilities $\chi_{2}$ and $\chi_{3}$ (oscillating with $2 \omega t$ and $3 \omega t$ ). The low signalto-noise ratio (in spite of gluing several single crystals) prevented us from obtaining good $\chi_{3}$ data; fortunately, we obtained nice curves for $\chi_{2}$, which is the leading nonlinear term when a bias field is applied.

The analysis and interpretation of the susceptibility curves were done with help from expressions derived with a simple system of balance equations (for the potential well populations). At variance with previous formulas, the field derivatives of the magnetic relaxation time are captured by our expressions. This, together with the known strong effect on $\tau\left(H_{z}\right)$ of resonant tunneling near the barrier top, permitted us to understand the experimental phenomenology. We also plugged into those equations the $\tau\left(H_{z}\right)$ obtained by solving a Pauli quantum master equation for $\mathrm{Mn}_{12}$, supporting this interpretation.
Near the resonant fields $H_{n}$ (matching the levels at both wells) the $\chi_{2}$ vs $H_{z}$ curves neatly amplify the resonant tunneling, as this entails large $d \tau / d H_{z}$. For fields between the $H_{n}$ 's, tunneling is blocked and the response is governed by the thermoactivation over the total barrier, as in the classical case. This does not give such a large $\tau^{\prime}$, while its sign is reversed with respect to the tunneling contribution. Thus the sensitivity of $\chi_{2}$ to the local features of the $\tau\left(H_{z}\right)$ curve provides an alternative method to assess if tunneling plays a role in the relaxation of a superparamagnet; and if so, in what field ranges it takes place.

\section{ACKNOWLEDGMENTS}

The work was funded by the DGA project PRONANOMAG PM011, DGES Projects No. MAT02-0166 and No. BFM2002-00113, and European Network of Excellence MAGMANet.
*Corresponding author. FAX: +34976761229. Email address: fluis@unizar.es

${ }^{1}$ D. Gatteschi and R. Sessoli, Angew. Chem., Int. Ed. 42, 268 (2003); S. J. Blundell and F. L. Pratt, J. Phys.: Condens. Matter 16, R771 (2004); B. Barbara, C. R. Phys. 6, 934 (2005).

${ }^{2}$ A. J. Leggett, J. Phys.: Condens. Matter 14, R415 (2002).

${ }^{3}$ J. L. van Hemmen and A. Süto, Physica B \& C 141, 37 (1986); M. Enz and R. Schilling, J. Phys. C 19, 1765 (1986); D. Garanin, J. Phys. A 24, L61 (1991).

${ }^{4}$ W. H. Zurek, Phys. Today 44(10), 36 (1991).

${ }^{5}$ C. Kiefer and E. Joos, in Quantum Future, edited by P. Blanchard and A. Jadczyk, Lecture Notes in Physics, Vol. 517 (Springer, Berlin, 1999), p. 105.

${ }^{6}$ J. R. Friedman, M. P. Sarachik, J. Tejada, and R. Ziolo, Phys. Rev. Lett. 76, 3830 (1996).

${ }^{7}$ J. M. Hernández, X. X. Zhang, F. Luis, J. Bartolomé, J. Tejada, and R. Ziolo, Europhys. Lett. 35, 301 (1996).

${ }^{8}$ L. Thomas, F. Lionti, R. Ballou, D. Gatteschi, R. Sessoli, and B. Barbara, Nature (London) 383, 145 (1996).
${ }^{9}$ W. Wernsdorfer, Adv. Chem. Phys. 118, 99 (2001).

${ }^{10}$ F. Luis, V. González, A. Millán, and J. L. García-Palacios, Phys. Rev. Lett. 92, 107201 (2004).

${ }^{11}$ R. López-Ruiz, F. Luis, V. González, A. Millán, and J. L. GarcíaPalacios, Phys. Rev. B 72, 224433 (2005).

${ }^{12}$ J. L. García-Palacios and P. Svedlindh, Phys. Rev. Lett. 85, 3724 (2000).

${ }^{13}$ J. L. García-Palacios and D. A. Garanin, Phys. Rev. B 70, 064415 (2004).

${ }^{14}$ W. F. Brown, Jr., Phys. Rev. 130, 1677 (1963).

${ }^{15}$ Y. L. Raikher, V. I. Stepanov, A. N. Grigorenko, and P. I. Nikitin, Phys. Rev. E 56, 6400 (1997).

${ }^{16}$ F. Luis, J. Bartolomé, and J. F. Fernández, Phys. Rev. B 57, 505 (1998).

${ }^{17}$ J. Villain, F. Hartmann-Boutron, R. Sessoli, and A. Rettori, Europhys. Lett. 27, 159 (1994).

${ }^{18}$ D. Zueco and J. L. García-Palacios, Phys. Rev. B 73, 104448 (2006). 